\title{
GENERALIZED INCREASING CONVEX AND DIRECTIONALLY CONVEX ORDERS
}

\author{
MICHEL M. DENUIT, ${ }^{*}$ Université Catholique de Louvain \\ MHAMED MESFIOUI,** Université du Québec à Trois-Rivières
}

\begin{abstract}
In this paper, the componentwise increasing convex order, the upper orthant order, the upper orthant convex order, and the increasing directionally convex order for random vectors are generalized to hierarchical classes of integral stochastic order relations. The elements of the generating classes of functions possess nonnegative partial derivatives up to some given degrees. Some properties of these new stochastic order relations are studied. Particular attention is paid to the comparison of weighted sums of the respective components of ordered random vectors. By providing a unified derivation of standard multivariate stochastic orderings, the present paper shows how some well-known results derive from a common principle.
\end{abstract}

Keywords: Integral stochastic order; supermodular function; directionally convex function

2000 Mathematics Subject Classification: Primary 60E15

\section{Introduction and motivation}

Stochastic orderings among random vectors have a wide field of applications in probability and statistics; see, e.g. Shaked and Shanthikumar (1994), (2007) and Denuit et al. (2005). Typically, these orders help to identify simpler, computationally more tractable, stochastic models, providing bounds on various quantities of interest.

In this paper we consider nonnegative random variables. Since all the order relations considered in this paper are invariant under shifts, all the results remain valid as long as the support of the random variables is bounded from below.

A number of stochastic orderings can be defined by reference to some class of measurable functions. Specifically, let $X$ and $Y$ be two random variables. Then, $X$ is said to be smaller than $Y$ in the ' ${ }_{*}$ ' ordering associated with the class $U_{*}^{[1]}$ of real-valued functions defined on (a subset of) the real line $\mathbb{R}$ if

$$
\mathrm{E}[g(X)] \leq \mathrm{E}[g(Y)] \quad \text { for all } g \in \mathcal{U}_{*}^{[1]},
$$

provided that the expectations exist. Whitt (1986) gave the name of integral stochastic orderings to the stochastic order relations defined by means of (1.1). The extension to $n$-dimensional random vectors $\boldsymbol{X}$ and $\boldsymbol{Y}$ is then clear: $\boldsymbol{X}$ is said to be smaller than $\boldsymbol{Y}$ in the ' $\preceq_{*}$ ' ordering associated with the class $\mathcal{U}_{*}^{[n]}$ of real-valued functions defined on (a subset of) the $n$-dimensional

Received 20 April 2009; revision received 16 November 2009.

* Postal address: Institut de Statistique and Institut des Sciences Actuarielles, Université Catholique de Louvain, B-1348 Louvain-la-Neuve, Belgium. Email address: michel.denuit@uclouvain.be

** Postal address: Département de Mathématiques et d'Informatique, Université du Québec à Trois-Rivières, TroisRivières (Québec), G9A 5H7 Canada. 
real space $\mathbb{R}^{n}$ if

$$
\mathrm{E}[g(\boldsymbol{X})] \leq \mathrm{E}[g(\boldsymbol{Y})] \text { for all } g \in \mathcal{U}_{*}^{[n]},
$$

provided that the expectations exist.

Among standard integral stochastic orderings used for comparing pairs of random variables, the stochastic dominance (denoted by ' $\preceq_{\text {st' }}$ ) is obtained from (1.1) when $U_{*}^{[1]}$ is the class of all the nondecreasing functions, and the increasing convex order (denoted by ' $\preceq_{\text {icx }}$ ') is obtained from (1.1) when $\mathcal{U}_{*}^{[1]}$ is the class of all the nondecreasing and convex functions. Corresponding orders for random vectors are obtained from (1.2).

In addition to these standard order relations, we also consider the univariate $s$-increasing convex orders, $s=1,2, \ldots$, introduced in Denuit et al. (1998) and defined as follows: given two random variables $X$ and $Y, X$ is said to be smaller than $Y$ in the $s$-increasing convex sense, denoted as $X \preceq_{s-\text { icx }} Y$, if (1.1) holds with $\mathcal{U}_{*}^{[1]}$ the class $U_{s-\text { icx }}^{[1]}$ of regular $s$-increasing convex functions $g$, i.e. those functions $g$ such that $\mathrm{d}^{k} g / \mathrm{d} x^{k} \geq 0$ for $k=1,2, \ldots, s$. Clearly, the ' $\preceq_{1-\mathrm{icx}}$ ' order is equivalent to the ' $\preceq_{\mathrm{st}}$ ' order and the ' $\preceq_{2-\mathrm{icx}}$ ' order is equivalent to the ' $\preceq_{\mathrm{icx}}$ ' order.

Let us now present some order relations for random vectors. To this end, let us denote by $F_{X}$ the joint distribution function of a random vector $X$ of dimension $n$ with components $X_{1}, X_{2}, \ldots, X_{n}$ and by $\bar{F}_{X}$ its multivariate survival function, that is,

$$
F_{\boldsymbol{X}}(\boldsymbol{x})=\operatorname{Pr}\left[X_{1} \leq x_{1}, X_{2} \leq x_{2}, \ldots, X_{n} \leq x_{n}\right]
$$

and

$$
\bar{F}_{\boldsymbol{X}}(\boldsymbol{x})=\operatorname{Pr}\left[X_{1}>x_{1}, X_{2}>x_{2}, \ldots, X_{n}>x_{n}\right] .
$$

Let $\boldsymbol{Y}$ be another $n$-dimensional random vector with distribution function $F_{\boldsymbol{Y}}$ and survival function $\bar{F}_{\boldsymbol{Y}}$. If the inequality

$$
\bar{F}_{X}(x) \leq \bar{F}_{Y}(x)
$$

is valid for all $\boldsymbol{x}$ then $\boldsymbol{X}$ is said to be smaller than $\boldsymbol{Y}$ in the upper orthant order (denoted by $\left.\boldsymbol{X} \preceq_{\text {uo }} \boldsymbol{Y}\right)$. The reason for this terminology is that sets of the form $\left\{\boldsymbol{x} \in \mathbb{R}^{n} \mid x_{1}>a_{1}, x_{2}>\right.$ $\left.a_{2}, \ldots, x_{n}>a_{n}\right\}$ for some fixed $\boldsymbol{a}$ are called upper orthants. Moreover, the relation ' $\preceq_{\mathrm{uo}}$ ' can be defined by means of (1.2) with $u_{*}^{[n]}$ the class $\underline{u}_{\text {uo }}$ of all the indicator functions for upper orthants. A general account of the properties of ' $\preceq$ uo' can be found in Shaked and Shanthikumar (2007, Section 6.G.1). Denoting as $\mathcal{U}_{\mathrm{uo}}^{\text {prod }}$ the class of all the products $\prod_{i=1}^{n} g_{i}$ where each $g_{i}: \mathbb{R}^{+} \rightarrow \mathbb{R}^{+}$is nondecreasing, it is well known that $\boldsymbol{X} \preceq$ uo $\boldsymbol{Y}$ if and only if (1.2) holds with $u_{*}^{[n]}=u_{\text {uo }}^{\text {prod }}$.

If $\boldsymbol{X} \preceq_{\mathrm{uo}} \boldsymbol{Y}$ holds then (1.2) in fact applies to a much larger class of functions than $\underline{u}_{\mathrm{uo}}$ or $u_{\mathrm{uo}}^{\mathrm{prod}}$. Specifically, define the forward difference operator $\Delta_{i, h_{i}}$ as

$$
\Delta_{i, h_{i}} g(\boldsymbol{x})=g\left(x_{1}, \ldots, x_{i-1}, x_{i}+h_{i}, x_{i+1}, \ldots, x_{n}\right)-g\left(x_{1}, \ldots, x_{n}\right)
$$

with $\Delta_{i, h_{i}}^{0} g(\boldsymbol{x})=g(\boldsymbol{x})$. Now, define $\overline{\mathcal{U}}_{\mathrm{uo}}$ as the class of all the functions $g$ such that

$$
\Delta_{1, h_{1}}^{k_{1}} \circ \cdots \circ \Delta_{n, h_{n}}^{k_{n}} g(\boldsymbol{x}) \geq 0
$$

for all $\boldsymbol{x}$, every $k_{1}, \ldots, k_{n} \in\{0,1\}$ such that $k_{1}+\cdots+k_{n} \geq 1$, and every $h_{1}, \ldots, h_{n} \geq 0$. Note that all the indicator functions of upper orthants belong to $\bar{u}_{\text {uo }}$, that is, $\underline{u}_{\text {uo }} \subset \bar{u}_{\text {uo. Indeed, }}$ we obtain, with $g(\boldsymbol{x})=\mathbb{I}\left[x_{1}>a_{1}, \ldots, x_{n}>a_{n}\right]$ for some fixed $\boldsymbol{a}$,

$$
\Delta_{1, h_{1}}^{k_{1}} \circ \cdots \circ \Delta_{n, h_{n}}^{k_{n}} g(\boldsymbol{x})=\prod_{i \mid k_{i}=0} \mathbb{I}\left[x_{i}>a_{i}\right] \prod_{i \mid k_{i}=1}\left(\mathbb{I}\left[x_{i}>a_{i}-h_{i}\right]-\mathbb{I}\left[x_{i}>a_{i}\right]\right) \geq 0 .
$$


Now, it can be shown that every $g \in \bar{U}_{\text {uo }}$ is the uniform limit of a sequence $g_{1}, g_{2}, \ldots$ of functions expressible as nonnegative linear combinations of indicator functions of upper orthants. It follows that $\boldsymbol{X} \preceq_{\mathrm{uo}} \boldsymbol{Y}$ if and only if (1.2) holds with $\mathcal{U}_{*}^{[n]}=\overline{\mathcal{U}}_{\mathrm{uo}}$.

Let us denote by $\mathcal{U}_{\mathrm{uo}}$ the class of all differentiable functions $g$ such that

$$
\frac{\partial^{k_{1}+\cdots+k_{n}}}{\partial x_{1}^{k_{1}} \cdots \partial x_{n}^{k_{n}}} g \geq 0
$$

for every integer $k_{1}, \ldots, k_{n} \in\{0,1\}$ such that $k_{1}+\cdots+k_{n} \geq 1$. It can be shown that $\mathcal{U}_{\text {uo }} \subset \bar{U}_{\text {uo. }}$ Since $U_{\text {uo }}$ is dense in $\bar{U}_{\text {uo }}$, then $\boldsymbol{X} \preceq$ uo $\boldsymbol{Y}$ if and only if (1.2) holds with $u_{*}^{[n]}=U_{\mathrm{uo}}$.

To sum up, we thus have the following equivalent characterizations of ' $\preceq$ uo'

$$
\begin{aligned}
X \preceq \text { uo } \boldsymbol{Y} & \Longleftrightarrow \quad(1.2) \text { holds with } \mathcal{U}_{*}^{[n]}=\underline{u}_{\mathrm{uo}} \\
& \Longleftrightarrow \quad(1.2) \text { holds with } U_{*}^{[n]}=\mathcal{u}_{\mathrm{uo}}^{\text {prod }} \\
& \Longleftrightarrow \quad \text { (1.2) holds with } U_{*}^{[n]}=\mathcal{u}_{\mathrm{uo}} \\
& \Longleftrightarrow \quad \text { (1.2) holds with } u_{*}^{[n]}=\bar{u}_{\mathrm{uo}} .
\end{aligned}
$$

This chain of equivalences allows us to identify rather 'small' generating classes of functions for ' $\preceq$ uo', the largest class as well as some intermediate classes. Small classes, such as $\underline{u}_{\mathrm{uo}}$, offer convenient ways to establish that a ' $\preceq$ uo' ranking indeed holds in some specific cases. Intermediate classes allow us to take advantage of particular features of the test functions, such as the differentiability for $U_{\mathrm{uo}}$ and the product form for $\mathcal{u}_{\mathrm{uo}}^{\mathrm{prod}}$. Finally, the maximal generator $\bar{U}_{\text {uo }}$ represents the largest class of functions such that the implication holds in (1.2). It is particularly useful in applications, once a ranking in the ' $\preceq_{\text {uo }}$ ' sense has been shown to hold.

When two random vectors $\boldsymbol{X}$ and $\boldsymbol{Y}$ are ordered with respect to some multivariate stochastic order relation, it is often interesting to know what kind of univariate order holds between the sums of the respective components of $\boldsymbol{X}$ and $\boldsymbol{Y}$, or between more general functions of these components. In this respect, Boutsikas and Vaggelatou (2002, Section 3.2) studied which stochastic order relation holds between the sums of the components of two random vectors ordered in the ' $\preceq$ uo' sense. They established that the following implication holds:

$$
\boldsymbol{X} \preceq_{\mathrm{uo}} \boldsymbol{Y} \Rightarrow \sum_{i=1}^{n} X_{i} \preceq_{n-\mathrm{icx}} \sum_{i=1}^{n} Y_{i} .
$$

Formula (1.3) indicates that a ' $\preceq_{\text {uo }}$ ' ordering between random vectors translates into an ' $\preceq_{n-\text { icx }}$ ' ordering between the sums of their respective components. The validity of (1.3) can easily be established as follows. For any function $g$ in $\mathcal{U}_{n-\mathrm{icx}}^{[1]}$, the function $\boldsymbol{x} \mapsto g\left(x_{1}+x_{2}+\cdots+x_{n}\right)$ clearly belongs to $\mathcal{U}_{\mathrm{uo}}$, whence the announced implication (1.3) follows.

Now, let us consider another multivariate stochastic order relation, close to ‘ $\preceq$ uo' and defined as follows. If the inequality

$$
\begin{aligned}
\int_{x_{1}}^{\infty} & \int_{x_{2}}^{\infty} \cdots \int_{x_{n}}^{\infty} \bar{F}_{\boldsymbol{X}}\left(u_{1}, u_{2}, \ldots, u_{n}\right) \mathrm{d} u_{1} \mathrm{~d} u_{2} \cdots \mathrm{d} u_{n} \\
& \leq \int_{x_{1}}^{\infty} \int_{x_{2}}^{\infty} \cdots \int_{x_{n}}^{\infty} \bar{F}_{\boldsymbol{Y}}\left(u_{1}, u_{2}, \ldots, u_{n}\right) \mathrm{d} u_{1} \mathrm{~d} u_{2} \cdots \mathrm{d} u_{n}
\end{aligned}
$$


holds true for all $\boldsymbol{x}$ then $\boldsymbol{X}$ is said to be smaller than $\boldsymbol{Y}$ in the upper orthant convex order (denoted by $\boldsymbol{X} \preceq_{\text {uocx }} \boldsymbol{Y}$ ). See Shaked and Shanthikumar (2007, Section 7.A.9). Denote by $\mathcal{U}_{\text {uocx }}^{\text {prod }}$ the class of all the products $\prod_{i=1}^{n} g_{i}$ where each $g_{i}: \mathbb{R}^{+} \rightarrow \mathbb{R}^{+}$is nondecreasing and convex. It is well known that $\boldsymbol{X} \preceq_{\text {uocx }} \boldsymbol{Y}$ if and only if (1.2) holds with $\boldsymbol{U}_{*}=\mathcal{U}_{\mathrm{uocx}}^{\text {prod }}$. Clearly, $\boldsymbol{X} \preceq_{\text {uo }} \boldsymbol{Y}$ implies that $\boldsymbol{X} \preceq$ uocx $\boldsymbol{Y}$.

Moreover, the two results recalled above for ' $\preceq_{\text {uo }}$ ' extend to ' $\preceq_{\text {uocx }}$ '. Specifically, denote by $u_{\text {uocx }}$ the class of all differentiable functions $g$ such that $\partial^{k_{1}+\cdots+k_{n}} g / \partial x_{1}^{k_{1}} \cdots \partial x_{n}^{k_{n}} \geq 0$ for every $k_{1}, \ldots, k_{n} \in\{0,1,2\}$ such that $k_{1}+\cdots+k_{n} \geq 1$. Then, $\boldsymbol{X} \preceq_{\text {uocx }} \boldsymbol{Y}$ if and only if (1.2) holds with $u_{*}=U_{\text {uocx }}$. Coming back to implication (1.3), it can be shown that the analogue for ' $\preceq$ uocx ' becomes

$$
\boldsymbol{X} \preceq_{\text {uocx }} \boldsymbol{Y} \Rightarrow \sum_{i=1}^{n} X_{i} \preceq_{2 n-\mathrm{icx}} \sum_{i=1}^{n} Y_{i} .
$$

Compared to (1.3), a ' $\preceq_{\text {uocx }}$ ' ordering between random vectors translates into a ' $2_{2 n-\text { icx }}$ ' ordering between the sums of their respective components.

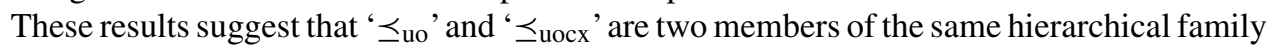
of stochastic order relations. We will show in Section 3 that this is indeed the case, and we will study some of the properties of this family. As ' $\preceq$ uo' can be characterized through (1.2) with $\mathcal{U}_{*}^{[n]}$ the class $\mathcal{U}_{\mathrm{uo}}^{\text {prod }}$ of products of elements in $\mathcal{U}_{1-\mathrm{icx}}^{[1]}$ and as ' $\preceq$ uocx' can be characterized through (1.2) with $\mathcal{U}_{*}^{[n]}$ the class $\mathcal{U}_{\mathrm{uocx}}^{\text {pod }}$ of products of elements in $\mathcal{U}_{2-\mathrm{icx}}^{[1]}$, it is natural to investigate the order relation resulting from products of elements in $u_{s_{i}-\text { icx }}^{[1]}$ for some positive integers $s_{i}$. This is done in Section 3 and it turns out that this hierarchical class of stochastic order relations inherits many of the properties of ' $\preceq$ uo' and ' $\preceq$ uocx'.

Of course, there are many other multivariate stochastic order relations for comparing pairs of random vectors. Suppose, for instance, that $\boldsymbol{X}$ and $\boldsymbol{Y}$ are such that (1.2) holds with $\mathcal{U}_{*}^{[n]}$ the class $U_{\text {iccx }}$ of all the nondecreasing functions $g$ that are convex in each argument when the other arguments are held fixed, provided that the expectations exist. Then $\boldsymbol{X}$ is said to be smaller than $\boldsymbol{Y}$ in the increasing componentwise convex order (denoted by $\boldsymbol{X} \preceq_{\text {iccx }} \boldsymbol{Y}$ ). See

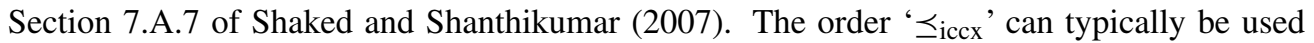
to compare random vectors with independent components. Specifically, let $X_{1}, X_{2}, \ldots, X_{n}$ be a set of independent random variables and let $Y_{1}, Y_{2}, \ldots, Y_{n}$ be another set of independent random variables. If $X_{i} \preceq_{\text {icx }} Y_{i}$ for $i=1,2, \ldots, n$ then $\boldsymbol{X} \preceq_{\text {iccx }} \boldsymbol{Y}$. The order resulting from the componentwise $s$-increasing convex functions is investigated in Section 2.

Another closely related stochastic order relation is the increasing directionally convex order. Recall that a function $g$ is said to be supermodular if the inequality $g(\boldsymbol{x})+g(\boldsymbol{y}) \leq g(\boldsymbol{x} \wedge \boldsymbol{y})+$ $g(\boldsymbol{x} \vee \boldsymbol{y})$ is valid for every $\boldsymbol{x}$ and $\boldsymbol{y}$, where the operators ' $\wedge$ ' and ' $\vee$ ' denote coordinatewise minimum and maximum, respectively. If $g$ has second-order partial derivatives then it is supermodular if and only if $\partial^{2} g / \partial x_{i} \partial x_{j} \geq 0$ for all $i \neq j$. Many examples of supermodular functions can be found in Chapter 6 of Marshall and Olkin (1979). Now, the function $g$ is said to be directionally convex if it is supermodular and coordinatewise convex. If $g$ is twice differentiable then it is directionally convex if and only if all its second derivatives are nonnegative.

If (1.2) holds with $\mathcal{U}_{*}^{[n]}$ the class $\mathcal{U}_{\text {idircx }}$ of all the nondecreasing directionally convex functions $g$ then $\boldsymbol{X}$ is said to be smaller than $\boldsymbol{Y}$ in the increasing directionally convex order (denoted by $\boldsymbol{X} \preceq_{\text {idircx }} \boldsymbol{Y}$ ). See Section 7.A.8 of Shaked and Shanthikumar (2007). Clearly, the chain of inclusions $U_{\text {uocx }} \subset U_{\text {idircx }} \subset U_{\text {iccx }}$ guarantees the chain of implications

$$
\boldsymbol{X} \preceq_{\text {iccx }} \boldsymbol{Y} \Rightarrow \boldsymbol{X} \preceq_{\text {idircx }} \boldsymbol{Y} \Rightarrow \boldsymbol{X} \preceq_{\text {uocx }} \boldsymbol{Y} \text {. }
$$




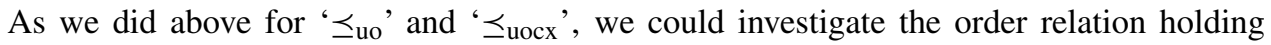
between the sum of the components of random vectors ordered in the ' $\preceq_{\text {idircx }}$ ' sense. In this case we obtain

$$
\boldsymbol{X} \preceq_{\text {idircx }} \boldsymbol{Y} \Rightarrow \sum_{i=1}^{n} X_{i} \preceq_{2-\text { icx }} \sum_{i=1}^{n} Y_{i} .
$$

The validity of (1.5) can easily be established as follows. For any function $g$ in $U_{2-\text { icx }}^{[1]}$, it is easily seen that the function $\boldsymbol{x} \mapsto g\left(x_{1}+x_{2}+\cdots+x_{n}\right)$ belongs to $\boldsymbol{U}_{\text {idircx }}$, whence the announced implication (1.5) follows. Compared to (1.3)-(1.4), we see that the degree of the order relation between $\sum_{i=1}^{n} X_{i}$ and $\sum_{i=1}^{n} Y_{i}$ does not depend on the dimension $n$ in (1.5).

Our aim in this paper is to embed ' $\preceq_{\mathrm{uo}}$ ', ' $\preceq_{\mathrm{uocx}}$ ', ' $\preceq_{\mathrm{iccx}}$ ', and ' $\preceq_{\text {idircx }}$ ' in three hierarchical classes of stochastic order relations:

- the multivariate $s$-increasing convex orders comprising ' $\mathrm{uoo}_{\mathrm{u}}$ ' and ' $\preceq_{\mathrm{uocx}}$ ' as special cases for $s=1$ and 2, respectively;

- the componentwise $s$-increasing convex orders extending ' $\preceq_{\text {iccx }}$ ', corresponding to $s=2$; and

- the $s$-increasing directionally convex orders generalizing ' $\preceq$ idircx', corresponding to $s=2$.

This will help us to understand the structure underlying these standard stochastic order relations, and also to realize the important role played by the number $n$ of dimensions relative to the degree $s$ of the orders. We propose a unified approach to defining ' iccx $_{\text {' }}$, ' $\preceq$ uo', ' $\preceq_{\text {uocx }}$ ', and ' $\preceq_{\text {idircx }}$ ' as integral stochastic orderings generated by classes of functions possessing nonnegative partial derivatives of specific degrees. This clearly shows how well-known results derive from a common principle and stresses the differences inherent to these standard multivariate stochastic orders.

After having discussed some of the properties of these classes of stochastic order relations, we compare positive linear combinations of the respective components of ordered random vectors. This allows us to extend (1.3)-(1.4) to the whole class of multivariate $s$-increasing convex orders, and (1.5) to the whole class of $s$-increasing directionally convex orders. In the former case, the dimension $n$ drives the degree of the resulting stochastic inequality between $\sum_{i=1}^{n} X_{i}$ and $\sum_{i=1}^{n} Y_{i}$, whereas in the latter case, this degree is not affected by $n$.

\section{Componentwise $s$-increasing convex order}

Let us define $U_{s-\text { iccx }}$ as the class of all functions $g$ that are $s$-increasing convex in each component, once the others are kept fixed. Then, we say that $\boldsymbol{X}$ is smaller than $\boldsymbol{Y}$ in the $s$-increasing componentwise convex order (denoted by $\boldsymbol{X} \preceq_{s-\text { iccx }} \boldsymbol{Y}$ ) if (1.2) holds with $U_{*}^{[n]}$ the class $u_{s-\text { iccx }}$. Clearly, the ' $\preceq_{2-\text { iccx }}$ ' order is equivalent to the ' $\preceq_{\text {iccx }}$ ' order and ' $\preceq_{1-i c c x}$ ' is the multivariate stochastic dominance ' $\preceq_{\mathrm{st}}$ '. The above definition can easily be extended by considering test functions $g$ that are $s_{i}$-increasing convex in the $i$ th component, once the others are kept fixed, $i=1,2, \ldots, n$.

As ' $\preceq_{\text {iccx }}$ ', the order ' $\preceq_{s-\text { iccx }}$ ' can typically be used to compare random vectors with independent components, as shown in the next result. The proof is rather standard and can be found in Shaked and Shanthikumar (2007, pp. 333-334) for the ' $\preceq_{\text {iccx }}$ ' order, so we do not repeat it here. It clearly shows why the ' $\preceq_{s-\text { iccx }}$ ' order is tailored to compare vectors with independent components. 
Property 2.1. Let $X_{1}, X_{2}, \ldots, X_{n}$ be a set of independent random variables, and let $Y_{1}$, $Y_{2}, \ldots, Y_{n}$ be another set of independent random variables. If $X_{i} \preceq_{s-\mathrm{icx}} Y_{i}$ for $i=1,2, \ldots, n$ then $\boldsymbol{X} \preceq_{s-i \operatorname{ccx}} \boldsymbol{Y}$.

\section{Multivariate $s$-increasing convex order}

Let $s=\left(s_{1}, \ldots, s_{n}\right)$ be a vector of positive integers. In view of the theory developed in the univariate case in Denuit et al. (1998) and in the bivariate case in Denuit et al. (1999), we say that the $n$-dimensional random vector $\boldsymbol{X}$ is smaller than the $n$-dimensional random vector $\boldsymbol{Y}$ in the $\boldsymbol{s}$-increasing convex order (denoted by $\boldsymbol{X} \preceq_{s-\text { icx }} \boldsymbol{Y}$ ) if (1.2) holds with $\mathcal{U}_{*}^{[n]}$ the class $u_{s-\text { icx }}$ of all the functions $g$ such that

$$
\frac{\partial^{k_{1}+k_{2}+\cdots+k_{n}}}{\partial x_{1}^{k_{1}} \partial x_{2}^{k_{2}} \cdots \partial x_{n}^{k_{n}}} g \geq 0 \text { for } k_{i}=0,1, \ldots, s_{i}, i=1,2, \ldots, n, k_{1}+k_{2}+\cdots+k_{n} \geq 1 .
$$

A particular case of interest is when $s_{1}=s_{2}=\cdots=s_{n}=s$, where we denote by ' ${ }_{s-\text { icx }}$ ' the resulting multivariate stochastic order relation.

This order can be characterized as follows.

Proposition 3.1. Let $\boldsymbol{X}$ and $\boldsymbol{Y}$ be two $n$-dimensional random vectors, and assume that $s_{i} \geq 1$ for $i=1,2, \ldots, n$. Then, $\boldsymbol{X} \preceq_{s-\mathrm{icx}} \boldsymbol{Y}$ if and only if (1.2) holds with $\mathcal{U}_{*}^{[n]}$ the class ${\underline{U_{s-\mathrm{icx}}}}_{\text {of }}$ all the functions of the form

$$
\prod_{i \in \mathcal{S}} x_{i}^{k_{i}} \prod_{j \in \overline{\mathcal{S}}}\left(x_{j}-t_{j}\right)_{+}^{s_{j}-1}
$$

where $S$ and $\bar{S}$ form a partition of $\{1,2, \ldots, n\}, k_{i}=0,1, \ldots, s_{i}-1$, and $t_{j} \geq 0$.

Proof. Consider $g \in U_{s-\text { icx }}$. By Taylor's expansion of $g$ viewed as a function of $x_{1}$ around 0 for fixed $x_{2}, \ldots, x_{n}$, we obtain

$$
g(\boldsymbol{x})=\sum_{i_{1}=0}^{s_{1}-1} \frac{\partial^{i_{1}} g\left(0, x_{2}, \ldots, x_{n}\right)}{\partial x_{1}^{i_{1}}} \frac{x_{1}^{i_{1}}}{i_{1} !}+\int_{0}^{+\infty} \frac{\left(x_{1}-t_{1}\right)_{+}^{s_{1}-1}}{\left(s_{1}-1\right) !} \frac{\partial^{s_{1}} g\left(t_{1}, x_{2}, \ldots, x_{n}\right)}{\partial x_{1}^{s_{1}}} \mathrm{~d} t_{1},
$$

and, by applying Taylor's expansion to $\partial^{i_{1}} g\left(0, x_{2}, \ldots, x_{n}\right) / \partial x_{1}^{i_{1}}$ and $\partial^{s_{1}} g\left(t_{1}, x_{2}, \ldots, x_{n}\right) / \partial x_{1}^{s_{1}}$ viewed as functions of $x_{2}$ around 0 , we obtain

$$
\begin{aligned}
g(\boldsymbol{x})= & \sum_{i_{1}=0}^{s_{1}-1} \sum_{i_{2}=0}^{s_{2}-1} \frac{\partial^{i_{1}+i_{2}} g\left(0,0, x_{3} \ldots, x_{n}\right)}{\partial x_{1}^{i_{1}} \partial x_{2}^{i_{2}}} \frac{x_{1}^{i_{1}}}{i_{1} !} \frac{x_{2}^{i_{2}}}{i_{2} !} \\
& +\sum_{i_{2}=0}^{s_{2}-1} \int_{0}^{+\infty} \frac{\left(x_{1}-t_{1}\right)_{+}^{s_{1}-1} x_{2}^{i_{2}}}{\left(s_{1}-1\right) ! i_{2} !} \frac{\partial^{s_{1}+i_{2}} g\left(t_{1}, 0, x_{3} \ldots, x_{n}\right)}{\partial x_{1}^{s_{1}} \partial x_{2}^{i_{2}}} \mathrm{~d} t_{1} \\
& +\sum_{i_{1}=0}^{s_{1}-1} \int_{0}^{+\infty} \frac{\left(x_{2}-t_{2}\right)_{+}^{s_{2}-1} x_{1}^{i_{1}}}{\left(s_{2}-1\right) ! i_{1} !} \frac{\partial^{i_{1}+s_{2}} g\left(0, t_{2}, x_{3} \ldots, x_{n}\right)}{\partial x_{1}^{i_{1}} \partial x_{2}^{s_{2}}} \mathrm{~d} t_{2} \\
& +\int_{0}^{+\infty} \int_{0}^{+\infty} \frac{\left(x_{1}-t_{1}\right)_{+}^{s_{1}-1}\left(x_{2}-t_{2}\right)_{+}^{s_{2}-1}}{\left(s_{1}-1\right) !\left(s_{2}-1\right) !} \frac{\partial^{s_{1}+s_{2}} g\left(t_{1}, t_{2}, x_{3} \ldots, x_{n}\right)}{\partial x_{1}^{s_{1}} \partial x_{2}^{s_{2}}} \mathrm{~d} t_{1} \mathrm{~d} t_{2} .
\end{aligned}
$$


By repeating this argument component by component, we obtain the next general expansion formula:

$$
\begin{aligned}
g(\boldsymbol{x})=\sum_{\mathcal{S}} \sum_{i \in \mathcal{S}} \sum_{k_{i}=0}^{s_{i}-1} \int_{0}^{+\infty} \cdots \int_{0}^{+\infty} & \prod_{i \in \mathcal{S}} \frac{x_{i}^{k_{i}}}{k_{i} !} \\
& \times \prod_{j \in \overline{\mathcal{S}}} \frac{\left(x_{j}-t_{j}\right)_{+}^{s_{j}-1}}{\left(s_{j}-1\right) !} \frac{\partial^{\sum_{i \in \mathcal{S}} k_{i}+\sum_{j \in \bar{S}} s_{j}}}{\prod_{i \in \mathcal{S}} \partial x_{i}^{k_{i}} \prod_{j \in \overline{\mathcal{S}}} \partial x_{j}^{s_{j}}} g\left(\boldsymbol{t}_{\overline{\mathcal{S}}}\right) \prod_{j \in \overline{\mathcal{S}}} \mathrm{d} t_{j},
\end{aligned}
$$

where $\boldsymbol{t}_{\bar{\delta}}=\sum_{i \in \overline{\mathcal{S}}} t_{i} \boldsymbol{e}_{i}$ and $\boldsymbol{e}_{i}=(0, \ldots, 0,1,0, \ldots, 0)$. It follows by Fubini's theorem that

$$
\begin{aligned}
\mathrm{E}[g(\boldsymbol{X})]=\sum_{\mathcal{S}} \sum_{i \in \mathcal{S}} \sum_{k_{i}=0}^{s_{i}-1} \int_{0}^{+\infty} \cdots \int_{0}^{+\infty} \frac{\mathrm{E}\left[\prod_{i \in \mathcal{S}} X_{i}^{k_{i}} \prod_{j \in \overline{\mathcal{S}}}\left(X_{j}-t_{j}\right)_{+}^{s_{j}-1}\right]}{\prod_{i \in \mathcal{S}} k_{i} ! \prod_{j \in \overline{\mathcal{S}}}\left(s_{j}-1\right) !} & \\
& \times \frac{\partial^{\sum_{i \in \mathcal{S}} k_{i}+\sum_{j \in \overline{\mathcal{S}}} s_{j}}}{\prod_{i \in \mathcal{S}} \partial x_{i}^{k_{i}} \prod_{j \in \overline{\mathcal{S}}} \partial x_{j}^{s_{j}}} g\left(\boldsymbol{t}_{\overline{\mathcal{S}}}\right) \prod_{j \in \overline{\mathcal{S}}} \mathrm{d} t_{j},
\end{aligned}
$$

which completes the proof.

Remark 3.1. Starting from $\bar{F}_{X}^{[1, \ldots, 1]}=\bar{F}_{\boldsymbol{X}}$, let us define the integrated right tails of $\boldsymbol{X}$ as

$$
\bar{F}_{\boldsymbol{X}}^{\left[k_{1}, \ldots, k_{i}+1, \ldots, k_{n}\right]}\left(x_{1}, \ldots, x_{i}, \ldots, x_{n}\right)=\int_{x_{i}}^{+\infty} \bar{F}_{\boldsymbol{X}}^{\left[k_{1}, \ldots, k_{i}, \ldots, k_{n}\right]}\left(x_{1}, \ldots, \xi_{i}, \ldots, x_{n}\right) \mathrm{d} \xi_{i} .
$$

It can be shown by induction that

$$
\bar{F}_{\boldsymbol{X}}^{[k]}(\boldsymbol{t})=\frac{\mathrm{E}\left[\prod_{i=1}^{n}\left(X_{i}-t_{i}\right)_{+}^{k_{i}-1}\right]}{\prod_{i=1}^{n}\left(k_{i}-1\right) !}
$$

Therefore, Proposition 3.1 can be equivalently expressed in terms of integrated right tails.

Let us now derive the largest class of functions for which the implication in (1.2) holds for ' $\preceq$ s-icx'.

Proposition 3.2. Let $\boldsymbol{X}$ and $\boldsymbol{Y}$ be two $n$-dimensional random vectors, and assume that $s_{i} \geq 1$ for $i=1,2, \ldots, n$. Then, $\boldsymbol{X} \preceq_{s-\mathrm{icx}} \boldsymbol{Y}$ if and only if (1.2) holds with $\mathcal{U}_{*}^{[n]}$ the class $\bar{u}_{\boldsymbol{s}-\mathrm{icx}}$ of all the functions $g$ such that

$$
\Delta_{1, h_{1}}^{k_{1}} \circ \cdots \circ \Delta_{n, h_{n}}^{k_{n}} g(\boldsymbol{x}) \geq 0 \quad \text { for all } \boldsymbol{x},
$$

for every $k_{i}=0,1, \ldots, s_{i}$ and $h_{1}, \ldots, h_{n} \geq 0$.

The next result is a direct consequence of Proposition 3.1. It ensures that we only have to consider the product of test functions to establish that ' $\preceq_{s-i c x}$ ' holds.

Proposition 3.3. Let $\boldsymbol{X}$ and $\boldsymbol{Y}$ be two $n$-dimensional random vectors. Then, $\boldsymbol{X} \preceq_{s-\mathrm{icx}} \boldsymbol{Y}$ if and only if (1.2) holds with $\mathcal{U}_{*}^{[n]}$ the class $\mathcal{U}_{\boldsymbol{s}-\mathrm{icx}}^{\mathrm{prod}}$ of all the products $\prod_{i=1}^{n} g_{i}$ where each $g_{i}: \mathbb{R}^{+} \rightarrow \mathbb{R}^{+}$belongs to $\mathcal{U}_{s_{i}-\mathrm{icx}}$. 
Recall that the $n$-dimensional random vector $\boldsymbol{X}$ is said to be positively upper orthant dependent (PUOD) when the inequality

$$
\operatorname{Pr}[\boldsymbol{X}>\boldsymbol{x}] \geq \prod_{i=1}^{n} \operatorname{Pr}\left[X_{i}>x_{i}\right] \text { holds for all } \boldsymbol{x} .
$$

If the reverse inequality holds in (3.1) then $X$ is said to be negatively upper orthant dependent (NUOD). Defining a random vector $\boldsymbol{X}^{\perp}$ with the same univariate marginals as $\boldsymbol{X}$ but with independent components, $\boldsymbol{X}$ is PUOD when $\boldsymbol{X}^{\perp} \preceq_{\text {uo }} \boldsymbol{X}$. We easily deduce from Proposition 3.1 that

$$
\boldsymbol{X}^{\perp} \preceq_{s-\mathrm{icx}} \boldsymbol{Y}^{\perp} \Longleftrightarrow X_{i} \preceq_{s_{i}-\mathrm{icx}} Y_{i} \quad \text { for } i=1,2, \ldots, n .
$$

This result can be strengthened as follows.

Property 3.1. (i) Let $\boldsymbol{X}$ be NUOD, and let $\boldsymbol{Y}$ have independent components. Then

$$
X_{i} \preceq_{s_{i}-\mathrm{icx}} Y_{i}, \quad i=1,2, \ldots, n \Longleftrightarrow \boldsymbol{X} \preceq_{s-\mathrm{icx}} \boldsymbol{Y} .
$$

(ii) Let $\boldsymbol{X}$ be PUOD, and let $\boldsymbol{Y}$ have independent components. Then

$$
Y_{i} \preceq_{s_{i}-\mathrm{icx}} X_{i}, \quad i=1,2, \ldots, n \quad \Longleftrightarrow \quad \boldsymbol{Y} \preceq_{s-\mathrm{icx}} \boldsymbol{X} .
$$

\section{The $s$-increasing directionally convex order}

In view of the definition of ' $\preceq$ idircx', we say that $\boldsymbol{X}$ is smaller than $\boldsymbol{Y}$ in the $s$-increasing directionally convex order (denoted by $\boldsymbol{X} \preceq_{s \text {-idircx }} \boldsymbol{Y}$ ) if (1.2) holds with $\boldsymbol{U}_{*}^{[n]}$ the class $\boldsymbol{U}_{s-\text { idircx }}$ of all the functions $g$ such that

$$
\frac{\partial^{k_{1}+k_{2}+\cdots+k_{n}}}{\partial x_{1}^{k_{1}} \partial x_{2}^{k_{2}} \cdots \partial x_{n}^{k_{n}}} g \geq 0
$$

for all the nonnegative integers $k_{1}, k_{2}, \ldots, k_{n}$ such that $1 \leq k_{1}+k_{2}+\cdots+k_{n} \leq s$.

For $s \geq n$, this new stochastic order relation is closely related to the higher-degree increasing convex order discussed in the preceding section. To see this, let us define $\mathcal{R}_{s}$ as the set of all the nonnegative integers $r_{1}, \ldots, r_{n}$ such that $1 \leq r_{1}+\cdots+r_{n}=s$. The following technical lemma relates $U_{s-\text { idircx }}$ to the classes of functions generating the $s$-increasing convex orders studied in the preceding section.

Lemma 4.1. If $s \geq n$ then the generator of ' ${ }_{s-\mathrm{idircx}}$ ' can be written in terms of the generators of the orderings ' ${ }_{\boldsymbol{r} \text {-icx }}$ ', $\boldsymbol{r} \in \mathcal{R}_{s}$, as follows:

$$
u_{s-\mathrm{idircx}}=\bigcap_{r \in \mathcal{R}_{s}} u_{r-\mathrm{icx}} .
$$

Proof. We first prove that $\mathcal{U}_{s-\text { idircx }} \subset \mathcal{U}_{\boldsymbol{r}-\mathrm{icx}}$ for all $\boldsymbol{r} \in \mathcal{R}_{s}$. To this end, let us consider $g \in \mathcal{U}_{s-\text { idircx }}$ and $\boldsymbol{r}=\left(r_{1}, \ldots, r_{n}\right) \in \mathcal{R}_{s}$. Now, for any $k_{1} \leq r_{1}, \ldots, k_{n} \leq r_{n}$ such that $1 \leq k_{1}+\cdots+k_{n}$, we have $1 \leq k_{1}+\cdots+k_{n} \leq r_{1}+\cdots+r_{n}=s$. Since $g \in U_{s-\text { idircx }}$, it then follows that

$$
\frac{\partial^{k_{1}+\cdots+k_{n}}}{\partial x_{1}^{k_{1}} \cdots \partial x_{n}^{k_{n}}} g \geq 0 \quad \text { for all such } k_{1}, \ldots, k_{n},
$$


which implies that $g \in \mathcal{U}_{\boldsymbol{r}-\mathrm{icx}}$ for all $\boldsymbol{r} \in \mathcal{R}_{s}$, and, thus, $g \in \bigcap_{\boldsymbol{r} \in \mathcal{R}_{s}} \mathcal{U}_{\boldsymbol{r}-\mathrm{icx}}$. Consequently, $u_{s-\text { idircx }} \subset \bigcap_{\boldsymbol{r} \in \mathcal{R}_{s}} u_{\boldsymbol{r}-\mathrm{icx}}$.

Now, consider $g \in \bigcap_{\boldsymbol{r} \in \mathcal{R}_{s}} U_{\boldsymbol{r}-\mathrm{icx}}$ and let $k_{1}, \ldots, k_{n}$ be such that $1 \leq k_{1}+\cdots+k_{n} \leq s$. We choose $r_{1}^{*}=k_{1}, \ldots, r_{n-1}^{*}=k_{n-1}$ and $r_{n}^{*}=s-\left(k_{1}+\cdots+k_{n-1}\right)$. By construction, $\boldsymbol{r}^{*}=$ $\left(r_{1}^{*}, \ldots, r_{n}^{*}\right) \in \mathcal{R}_{s}$ with $k_{1}=r_{1}^{*}, \ldots, r_{n-1}^{*}=k_{n-1}$ and $k_{n} \leq r_{n}^{*}$. Since $g \in \bigcap_{\boldsymbol{r} \in \mathcal{R}_{s}} u_{\boldsymbol{r}-\mathrm{icx}}$, we have in particular that $g \in U_{r^{*}-\mathrm{icx}}$. Hence,

$$
\frac{\partial^{k_{1}+\cdots+k_{n}}}{\partial x_{1}^{k_{1}} \cdots \partial x_{n}^{k_{n}}} g \geq 0 \quad \text { for all such } k_{1}, \ldots, k_{n},
$$

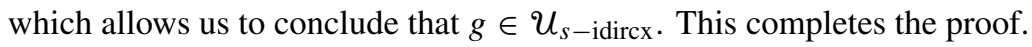

Now, let us define the following classes of functions:

$$
\begin{aligned}
& \underline{u}_{s-\text { idircx }}=\bigcap_{r \in \mathcal{R}_{s}} \underline{u}_{r-\mathrm{icx}}, \\
& \bar{u}_{s-\mathrm{idircx}}=\bigcap_{\boldsymbol{r} \in \mathcal{R}_{s}} \bar{u}_{\boldsymbol{r}-\mathrm{icx}}, \\
& u_{s-\text { idircx }}^{\text {prod }}=\bigcap_{\boldsymbol{r} \in \mathcal{R}_{s}} u_{\boldsymbol{r}-\mathrm{icx}}^{\text {prod }} .
\end{aligned}
$$

We are in a position to state the following characterizations of ' $\varliminf_{s-\text { idircx' }}$.

Proposition 4.1. Let $\boldsymbol{X}$ and $\boldsymbol{Y}$ be two $n$-dimensional random vectors, and assume that $s \geq n$. Then,

$$
\begin{aligned}
X \preceq_{s-\operatorname{idircx}} \boldsymbol{Y} & \Longleftrightarrow \quad \text { (1.2) holds with } u_{*}^{[n]}=\underline{U}_{s-\operatorname{idircx}} \\
& \Longleftrightarrow \quad \text { (1.2) holds with } u_{*}^{[n]}=\bar{U}_{s-\operatorname{idircx}} \\
& \Longleftrightarrow \quad \text { (1.2) holds with } u_{*}^{[n]}=u_{s-\text { idircx }}^{\text {prod }}
\end{aligned}
$$

Remark 4.1. Note that the condition $s \geq n$ is necessary to obtain characterizations of the stochastic ordering ' $\preceq_{s-\text { idircx' }}$. When $s<n$, it is not possible to obtain a dense subclass for the generating class $U_{s-\text { idircx }}$ of this ordering. This explains why in dimension 3 the supermodular order does not possess any characterization involving subclasses of the (regular) supermodular functions. If $s<n$, we face the same problem as with the supermodular order. In dimension 2 , this order coincides with the ' $\preceq$ uo' order (restricted to random couples with identical univariate marginals) and can be characterized as explained in the introduction. For dimensions 3 and over, there is no dense subclass of the supermodular functions that can be used to characterize this integral stochastic order.

\section{Properties}

We now establish various properties of the stochastic order relations introduced above. Firstly, as $U_{t-\text { icx }} \subset U_{s-\text { icx }}$ provided $t \geq s$, that is, $t_{i} \geq s_{i}$ for all $i=1,2, \ldots, n$, and $u_{t-\text { idircx }} \subset U_{s-\text { idircx }}$ for all integers $t \geq s$, we deduce that the $s$-increasing convex and $s$-increasing directionally convex orders form hierarchical classes of stochastic order relations, i.e. stochastic inequalities weaken as the degree of the order increases. This is formally stated in the next result. 
Property 5.1. For any $\boldsymbol{t} \geq \boldsymbol{s}$, we have $\boldsymbol{X} \preceq_{\boldsymbol{s}-\mathrm{icx}} \boldsymbol{Y}$ implies that $\boldsymbol{X} \preceq_{\boldsymbol{t}-\mathrm{icx}} \boldsymbol{Y}$. Similarly, for any $t \geq s$, we have $\boldsymbol{X} \preceq_{s-\operatorname{idircx}} \boldsymbol{Y}$ implies that $\boldsymbol{X} \preceq_{t-\operatorname{idircx}} \boldsymbol{Y}$.

These stochastic orders are clearly closed under mixtures. This is stated in the next property, together with some direct consequences involving sums and other functions of random vectors.

Property 5.2. (i) Let $\boldsymbol{X}$ and $\boldsymbol{Y}$ be two n-dimensional random vectors, and let $\boldsymbol{\Theta}$ be an $m$-dimensional random vector. Then,

$$
[\boldsymbol{X} \mid \boldsymbol{\Theta}=\boldsymbol{\theta}] \preceq_{\boldsymbol{s}-\mathrm{icx}}[\boldsymbol{Y} \mid \boldsymbol{\Theta}=\boldsymbol{\theta}] \text { for all } \boldsymbol{\theta} \Rightarrow \boldsymbol{X} \preceq_{\boldsymbol{s}-\mathrm{icx}} \boldsymbol{Y}
$$

and

$$
[\boldsymbol{X} \mid \boldsymbol{\Theta}=\boldsymbol{\theta}] \preceq_{s-\operatorname{idircx}}[\boldsymbol{Y} \mid \boldsymbol{\Theta}=\boldsymbol{\theta}] \text { for all } \boldsymbol{\theta} \Rightarrow \boldsymbol{X} \preceq_{s-\operatorname{idircx}} \boldsymbol{Y} .
$$

(ii) Let $\boldsymbol{X}$ and $\boldsymbol{Y}$ be two $n$-dimensional random vectors such that $\boldsymbol{X} \preceq_{s-\mathrm{icx}} \boldsymbol{Y}$, and let $\boldsymbol{Z}$ be an $m$-dimensional random vector, which is independent from $\boldsymbol{X}$ and $\boldsymbol{Y}$. Then,

$$
\boldsymbol{X} \preceq_{\boldsymbol{s}-\mathrm{icx}} \boldsymbol{Y} \Rightarrow\left(\psi_{1}\left(X_{1}, \boldsymbol{Z}\right), \ldots, \psi_{n}\left(X_{n}, \boldsymbol{Z}\right)\right) \preceq_{\boldsymbol{s}-\mathrm{icx}}\left(\psi_{1}\left(Y_{1}, \boldsymbol{Z}\right), \ldots, \psi_{n}\left(Y_{n}, \boldsymbol{Z}\right)\right)
$$

whenever $\psi_{i}(x, z), i=1,2, \ldots, n$, viewed as a functions of $x$, with $z$ fixed, is $s_{i}$-increasing convex for any $\boldsymbol{z}$. In particular, consider the $n$-dimensional random vectors $\boldsymbol{X}, \boldsymbol{Y}$, and $\boldsymbol{Z}$ such that $\boldsymbol{Z}$ is independent from both $\boldsymbol{X}$ and $\boldsymbol{Y}$. Then,

$$
X \preceq_{s-\mathrm{icx}} \boldsymbol{Y} \Rightarrow \boldsymbol{X}+\boldsymbol{Z} \preceq_{s-\mathrm{icx}} \boldsymbol{Y}+\boldsymbol{Z}
$$

and

$$
\boldsymbol{X} \preceq_{s-\operatorname{idircx}} \boldsymbol{Y} \Rightarrow \boldsymbol{X}+\boldsymbol{Z} \preceq_{s-\operatorname{idircx}} \boldsymbol{Y}+\boldsymbol{Z} .
$$

(iii) Consider two sets of independent $n$-dimensional random vectors, $\boldsymbol{X}_{1}, \boldsymbol{X}_{2}, \ldots, \boldsymbol{X}_{m}$ and $\boldsymbol{Y}_{1}, \boldsymbol{Y}_{2}, \ldots, \boldsymbol{Y}_{m}$, say. Then,

$$
\boldsymbol{X}_{i} \preceq_{s-\mathrm{icx}} \boldsymbol{Y}_{i} \quad \text { for } i=1,2, \ldots, m \Rightarrow \sum_{i=1}^{m} \boldsymbol{X}_{i} \preceq_{s-\mathrm{icx}} \sum_{i=1}^{m} \boldsymbol{Y}_{i}
$$

and

$$
\boldsymbol{X}_{i} \preceq_{s-\operatorname{idircx}} \boldsymbol{Y}_{i} \quad \text { for } i=1,2, \ldots, m \Rightarrow \sum_{i=1}^{m} \boldsymbol{X}_{i} \preceq_{s-\operatorname{idircx}} \sum_{i=1}^{m} \boldsymbol{Y}_{i} .
$$

(iv) Consider two sequences of independent $n$-dimensional random vectors, $\boldsymbol{X}_{1}, \boldsymbol{X}_{2}, \ldots$ and $\boldsymbol{Y}_{1}, \boldsymbol{Y}_{2}, \ldots$, say, and the positive integer-valued random variable $N$, independent from the $\boldsymbol{X}_{i} \boldsymbol{s}$ and the $\boldsymbol{Y}_{j}$ s. Then,

$$
\boldsymbol{X}_{i} \preceq_{s-\mathrm{icx}} \boldsymbol{Y}_{i} \text { for all } i \Rightarrow \sum_{i=1}^{N} \boldsymbol{X}_{i} \preceq_{s-\mathrm{icx}} \sum_{i=1}^{N} \boldsymbol{Y}_{i}
$$

and

$$
\boldsymbol{X}_{i} \preceq_{s-\text { idircx }} \boldsymbol{Y}_{i} \text { for all } i \Rightarrow \sum_{i=1}^{N} \boldsymbol{X}_{i} \preceq_{s-\text { idircx }} \sum_{i=1}^{N} \boldsymbol{Y}_{i}
$$


Aggregating independent random variables ordered in the univariate $s$-increasing convex sense produces random vectors ordered in both the ' $\preceq_{s-\text { icx }}$ ' sense and ' $\preceq_{s-\text { idircx }}$ ' sense. This comes from the fact that ' $\preceq_{s-\text { iccx }}$ ' implies both ' $\preceq_{s-\text { icx }}$ ' and ' $\preceq_{s-\text { idircx' }}$. This result stays valid when blocks of random variables are concatenated, as shown next. Note that we take $s_{1}=\cdots=s_{n}=s$ in this case.

Property 5.3. Let $\boldsymbol{X}_{1}, \boldsymbol{X}_{2}, \ldots, \boldsymbol{X}_{m}$ be independent $k_{i}$-dimensional random vectors, where all the $k_{i}$ s are greater than or equal to 2 . Let $\boldsymbol{Y}_{1}, \boldsymbol{Y}_{2}, \ldots, \boldsymbol{Y}_{m}$ be another set of independent $k_{i}$-dimensional random vectors. If $\boldsymbol{X}_{i} \preceq_{s-\mathrm{icx}} \boldsymbol{Y}_{i}$ for $i=1,2, \ldots, m$ then

$$
\left(\boldsymbol{X}_{1}, \boldsymbol{X}_{2}, \ldots, \boldsymbol{X}_{m}\right) \preceq_{s-\mathrm{icx}}\left(\boldsymbol{Y}_{1}, \boldsymbol{Y}_{2}, \ldots, \boldsymbol{Y}_{m}\right) .
$$

Similarly, if $\boldsymbol{X}_{i} \preceq_{s-\operatorname{idircx}} \boldsymbol{Y}_{i}$ for $i=1,2, \ldots$, m then

$$
\left(\boldsymbol{X}_{1}, \boldsymbol{X}_{2}, \ldots, \boldsymbol{X}_{m}\right) \preceq_{s-\operatorname{idircx}}\left(\boldsymbol{Y}_{1}, \boldsymbol{Y}_{2}, \ldots, \boldsymbol{Y}_{m}\right) .
$$

Proof. Let $\boldsymbol{X}_{1}$ and $\boldsymbol{X}_{2}$ be two independent $k_{1}$ - and $k_{2}$-dimensional random vectors, and let $\boldsymbol{Y}_{1}$ and $\boldsymbol{Y}_{2}$ be two other independent $k_{1}$ - and $k_{2}$-dimensional random vectors. Suppose that $\boldsymbol{X}_{1} \preceq_{s-\text { icx }} \boldsymbol{Y}_{1}$ and $\boldsymbol{X}_{2} \preceq_{s-\text { icx }} \boldsymbol{Y}_{2}$. Then, for any $g \in U_{s-\text { icx }}$, we have

$$
\begin{aligned}
\mathrm{E}\left[g\left(\boldsymbol{X}_{1}, \boldsymbol{X}_{2}\right)\right] & =\mathrm{E}\left[\mathrm{E}\left[g\left(\boldsymbol{X}_{1}, \boldsymbol{X}_{2}\right) \mid \boldsymbol{X}_{2}\right]\right] \\
& \leq \mathrm{E}\left[\mathrm{E}\left[g\left(\boldsymbol{Y}_{1}, \boldsymbol{X}_{2}\right) \mid \boldsymbol{X}_{2}\right]\right] \\
& =\mathrm{E}\left[g\left(\boldsymbol{Y}_{1}, \boldsymbol{X}_{2}\right)\right] \\
& \leq \mathrm{E}\left[g\left(\boldsymbol{Y}_{1}, \boldsymbol{Y}_{2}\right)\right],
\end{aligned}
$$

where the first inequality follows from the fact that $g\left(\boldsymbol{x}_{1}, \boldsymbol{x}_{2}\right)$ viewed as a function of $\boldsymbol{x}_{1}$, with $\boldsymbol{x}_{2}$ fixed, is $s$-increasing convex, and the second inequality follows in a similar manner. The announced result then follows from the above by mathematical induction.

If we fix the values of some arguments of an $s$-increasing convex or of an $s$-increasing directionally convex function, we still have an $s$-increasing convex or an $s$-increasing directionally convex function. This ensures that the integral stochastic order relations generated by these classes of functions are closed under marginalization, as formally stated in the next result.

Property 5.4. Let $\boldsymbol{X}$ and $\boldsymbol{Y}$ be two $n$-dimensional random vectors. If $\boldsymbol{X} \preceq_{s-\mathrm{icx}} \boldsymbol{Y}$ then, for any $\tau \subseteq\{1,2, \ldots, n\}$,

$$
\left(X_{i}\right)_{i \in \mathcal{I}} \preceq_{s-\mathrm{icx}}\left(Y_{i}\right)_{i \in \mathcal{I}},
$$

where $\left(X_{i}\right)_{i \in \mathcal{I}}$ denotes the \# $\mathcal{I}$-dimensional random vector formed with the components of $\boldsymbol{X}$ with indices in $\mathcal{I}$. Similarly, if $\boldsymbol{X} \preceq_{s-\text { idircx }} \boldsymbol{Y}$ then, for any $\mathcal{I} \subseteq\{1,2, \ldots, n\}$ with $\# \mathcal{I}=p \geq 2$,

$$
\left(X_{i}\right)_{i \in \mathcal{X}} \preceq_{s-\operatorname{idircx}}\left(Y_{i}\right)_{i \in \mathcal{X}} .
$$

Moreover, $X_{i} \preceq_{s-\mathrm{icx}} Y_{i}$ when $\mathcal{I}=\{i\}$.

\section{Comparison of univariate transforms, with special emphasis on nonnegative linear combinations}

Let us now consider the relationship between multivariate stochastic order relations and univariate stochastic dominance between positive linear combinations of their respective components. Having two ordered random vectors $\boldsymbol{X}$ and $\boldsymbol{Y}$, we would like to investigate the degree of the stochastic dominance relation holding between $\sum_{i=1}^{n} \alpha_{i} X_{i}$ and $\sum_{i=1}^{n} \alpha_{i} Y_{i}$ with nonnegative $\alpha_{1}, \ldots, \alpha_{n}$. 
Property 6.1. (i) Consider a nonnegative function $\Psi$ in $\mathcal{U}_{\boldsymbol{s}-\mathrm{icx}}$. Then,

$$
\boldsymbol{X} \preceq_{s-\mathrm{icx}} \boldsymbol{Y} \Rightarrow \Psi(\boldsymbol{X}) \preceq_{\left(\sum_{i=1}^{n} s_{i}\right)-\mathrm{icx}} \Psi(\boldsymbol{Y}) .
$$

In particular,

$$
\boldsymbol{X} \preceq_{s-\mathrm{icx}} \boldsymbol{Y} \Rightarrow \sum_{i=1}^{n} \alpha_{i} X_{i} \preceq_{\left(\sum_{i=1}^{n} s_{i}\right)-\mathrm{icx}} \sum_{i=1}^{n} \alpha_{i} Y_{i} \quad \text { for any } \alpha_{1}, \ldots, \alpha_{n} \geq 0 .
$$

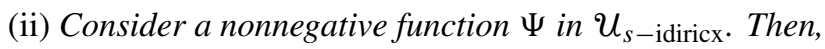

$$
\boldsymbol{X} \preceq_{s-\operatorname{idircx}} \boldsymbol{Y} \Rightarrow \Psi(\boldsymbol{X}) \preceq_{s-\mathrm{icx}} \Psi(\boldsymbol{Y}) .
$$

In particular,

$$
\boldsymbol{X} \preceq_{s-\operatorname{idircx}} \boldsymbol{Y} \Rightarrow \sum_{i=1}^{n} \alpha_{i} X_{i} \preceq_{s-\mathrm{icx}} \sum_{i=1}^{n} \alpha_{i} Y_{i} \quad \text { for any } \alpha_{1}, \ldots, \alpha_{n} \geq 0 .
$$

Proof. To establish (i), let us consider $g$ in $U_{\left(\sum_{i=1}^{n} s_{i}\right)-\text { icx }}$. Then, it is easily seen that $g \circ \Psi$ belongs to $U_{\boldsymbol{s}-\mathrm{icx}}$, so that $\boldsymbol{X} \preceq_{s-\mathrm{icx}} \boldsymbol{Y}$ ensures that the stochastic inequality $\Psi(\boldsymbol{X}) \preceq_{\left(\sum_{i=1}^{n} s_{i}\right)-\mathrm{icx}}$ $\Psi(\boldsymbol{Y})$ holds between the random variables $\Psi(\boldsymbol{X})$ and $\Psi(\boldsymbol{Y})$. Considering $\Psi(\boldsymbol{x})=\sum_{i=1}^{n} \alpha_{i} x_{i}$ with $\alpha_{1}, \ldots, \alpha_{n} \geq 0$, we clearly see that $\Psi \in \mathcal{U}_{s-\mathrm{icx}}$. The proof of (ii) is similar.

It is even possible to obtain order relations for sums of functions of the components of the respective random vectors. Indeed, since

$$
\boldsymbol{X} \preceq_{s-\mathrm{icx}} \boldsymbol{Y} \Rightarrow\left(\psi_{1}\left(X_{1}\right), \psi_{2}\left(X_{2}\right), \ldots, \psi_{n}\left(X_{n}\right)\right) \preceq_{s-\mathrm{icx}}\left(\psi_{1}\left(Y_{1}\right), \psi_{2}\left(Y_{2}\right), \ldots, \psi_{n}\left(Y_{n}\right)\right)
$$

for all nonnegative $\psi_{i} \in \mathcal{U}_{s_{i}-\mathrm{icx}}$, we also have

$$
\boldsymbol{X} \preceq_{s-\mathrm{icx}} \boldsymbol{Y} \Rightarrow \sum_{i=1}^{n} \psi_{i}\left(X_{i}\right) \preceq_{\left(\sum_{i=1}^{n} s_{i}\right)-\mathrm{icx}} \sum_{i=1}^{n} \psi_{i}\left(Y_{i}\right) .
$$

Similarly, as

$$
\boldsymbol{X} \preceq_{s-\operatorname{idircx}} \boldsymbol{Y} \Rightarrow\left(\psi_{1}\left(X_{1}\right), \psi_{2}\left(X_{2}\right), \ldots, \psi_{n}\left(X_{n}\right)\right) \preceq_{s-\operatorname{idircx}}\left(\psi_{1}\left(Y_{1}\right), \psi_{2}\left(Y_{2}\right), \ldots, \psi_{n}\left(Y_{n}\right)\right)
$$

for all nonnegative $\psi_{i} \in U_{s-\text { icx }}$, we also have

$$
\boldsymbol{X} \preceq_{s-\operatorname{idircx}} \boldsymbol{Y} \Rightarrow \sum_{i=1}^{n} \psi_{i}\left(X_{i}\right) \preceq_{s-\mathrm{icx}} \sum_{i=1}^{n} \psi_{i}\left(Y_{i}\right) .
$$

\section{Acknowledgements}

Michel Denuit acknowledges the financial support of the Communautéfrançaise de Belgique under contract 'Projet d'Actions de Recherche Concertées' ARC 04/09-320, of the Banque Nationale de Belgique under grant 'Risk measures and Economic capital', and of the Onderzoeksfonds K.U. Leuven (GOA/07: Risk Modeling and Valuation of Insurance and Financial Cash Flows, with Applications to Pricing, Provisioning and Solvency).

Mhamed Mesfioui acknowledges the financial support of the Natural Sciences and Engineering Research Council of Canada. 


\section{References}

Boutsikas, M. V. and Vaggelatou, E. (2002). On the distance between convex-ordered random variables, with applications. Adv. Appl. Prob. 34, 349-374.

Denuit, M., Lefèvre, C. AND Mesfioui, M. (1999). A class of bivariate stochastic orderings, with applications in actuarial sciences. Insurance Math. Econom. 24, 31-50.

Denuit, M., LefÈvre, C. And ShaKed, M. (1998). The s-convex orders among real random variables, with applications. Math. Inequal. Appl. 1, 585-613.

Denuit, M., Dhaene, J., Goovaerts, M. J. and KaAs, R. (2005). Actuarial Theory for Dependent Risks: Measures, Orders and Models. John Wiley, New York.

Marshall, A. W. And Olkin, I. (1979). Inequalities: Theory of Majorization and Its Applications. Academic Press, New York.

Shaked, M. And Shanthikumar, J. G. (1994). Stochastic Orders and Their Applications. Academic Press, Boston, MA.

Shaked, M. and Shanthikumar, J. G. (2007). Stochastic Orders. Springer, New York.

WhITT, W. (1986). Stochastic comparisons for non-Markov processes. Math. Operat. Res. 11, 609-618. 